\title{
Isotermas de dessorção das sementes de nabo forrageiro obtidas pelos métodos dinâmico e estático
}

\author{
Kelly A. de Sousa ${ }^{1}$, Osvaldo Resende ${ }^{1} \&$ Lílian M. Costa ${ }^{1}$
}

\begin{abstract}
RESUMO
Propôs-se, com este trabalho, determinar as isotermas de dessorção das sementes de nabo forrageiro e ajustar modelos matemáticos aos dados experimentais obtidos pelos métodos estático e dinâmico e verificar a possibilidade de utilização de um único modelo para ambos os métodos. Os teores de água de equilíbrio foram determinados pelo método dinâmico para as temperaturas de $25,30,35$ e $40{ }^{\circ} \mathrm{C}$ e atividades de água para cada temperatura, entre 0,27 a 0,82. No método estático foram utilizados dessecadores contendo as amostras e soluções salinas saturadas que foram colocadas em câmaras B.O.D. mantidas nas mesmas temperaturas. Aos dados experimentais foram ajustados modelos matemáticos disponíveis na literatura. Segundo os parâmetros estatísticos de análise, o modelo de Copace foi o que descreveu melhor a higroscopicidade das sementes de nabo forrageiro obtida pelo método dinâmico e, para o método estático, os modelos de Sigma Copace, Oswin, Halsey Modificado e Copace foram os que melhor representaram a higroscopicidade das sementes de nabo forrageiro. Segundo a técnica de identidade de modelos, é possível ajustar uma única equação de Copace aos dados experimentais para os dois métodos analisados.
\end{abstract}

Palavras-chave: oleaginosa, equilíbrio higroscópico, modelagem matemática, identidade de modelos

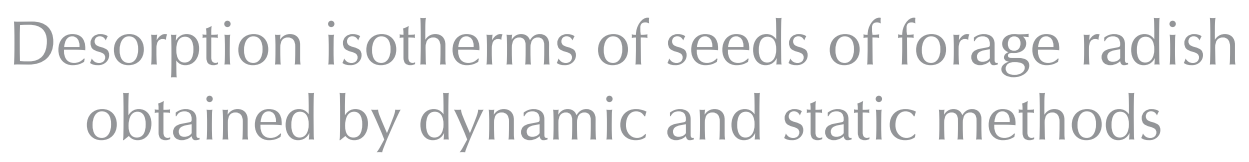

\begin{abstract}
This study aimed to determine the desorption isotherms of radish seeds and adjust mathematical models to experimental data obtained by static and dynamic methods, and to verify the possibility of using a single model for both methods. The equilibrium moisture contents were determined by dynamic method at temperatures of $25,30,35$, and $40{ }^{\circ} \mathrm{C}$ and water activity for each temperature from 0.27 to 0.82 . In the static method desiccators were used containing saturated salt solutions and samples that were placed in B.O.D (Biological Oxygen Demand) chambers maintained at the same temperatures. Mathematical models available in the literature were adjusted to experimental data. According to statistical analysis parameters, Copace model was the one that best described the hygroscopicity of radish seeds obtained by the dynamic method; for the static models, Copace Sigma, Oswin, Modified Halsey and Copace were the ones that best represented hygroscopicity of radish seeds. According to the technique of model identity, it is possible to adjust a single Copace equation to the experimental data for both analyzed methods.
\end{abstract}

Key words: oleaginous, hygroscopic equilibrium, mathematical modeling, model identity 


\section{INTRODUÇÃO}

Tendo em vista a crescente demanda de alternativas aos combustíveis de origem fóssil, diversas plantas vêm sendo estudadas com objetivo de fornecimento de óleo para produção de biocombustíveis. Entre essas plantas o nabo forrageiro vem surgindo como matéria-prima potencial para obtenção de biocombustível visto que suas sementes apresentam teores de óleo na faixa de 40 a 54\% (Domingos, 2005).

O nabo forrageiro (Raphanus sativus L.) pertencente à família das brassicaceas, está entre as mais antigas espécies usadas para a produção de óleo vegetal, sendo cultivado principalmente na Ásia Oriental. Além de ser utilizado na extração de óleo para a produção de biodiesel, é empregado para adubação verde e rotação de culturas (Derpsch \& Calegari, 1992) e tem sido empregada nas regiões sul, sudeste e centrooeste do Brasil, como adubo verde de inverno e como planta de cobertura, para proteger o solo contra erosões (Crusciol et al., 2005).

As sementes das brassicaceas são materiais higroscópicos, ou seja, são capazes de absorver, reter ou eliminar água e procuram estabelecer, sempre, um equilíbrio de umidade com o ar em seu redor. Em geral, a água causa aumento da pressão de vapor sobre a superfície dos grãos. Quando a pressão de vapor da água presente no produto e a pressão de vapor de água da atmosfera são iguais, ocorre o equilíbrio higroscópico (Araújo et al., 2005).

A relação entre o teor de água de equilíbrio e a umidade relativa de equilíbrio, também designada por atividade da água, constitui fator essencial nos projetos e estudos de sistemas de secagem, manuseio, armazenagem, embalagem e transporte e na modelagem da longevidade das sementes (Araújo et al., 2001).

Para Hall (1980) as curvas de equilíbrio higroscópico são importantes para definir limites de desidratação do produto, estimar as mudanças do teor de água sob determinada condição de temperatura e umidade relativa do ambiente e para definir os teores de água adequados ao início de atividade de microorganismos, que podem provocar deterioração do produto. A composição química do produto influencia diretamente o processo de sorção de água. Segundo Brooker et al. (1992) grãos com elevado teor de óleo adsorvem menor quantidade de água do ambiente do que os grãos com alto teor de amido.

As curvas de teor de água de equilíbrio podem ser obtidas experimentalmente por meio dos métodos dinâmico e estático (Corrêa et al., 2005a).

No método dinâmico o grão é submetido a fluxos de ar sob condições controladas de temperatura e umidade relativa até que seja atingido o equilíbrio. No método estático o equilíbrio higroscópico entre o produto e o ambiente sob condições controladas é atingido sem movimentação do ar (Chen, 2000; Côrrea et al., 2005a).

O comportamento higroscópico de diversos produtos agrícolas tem sido estudado por vários pesquisadores, que descrevem modelos distintos para expressar o teor de água de equilíbrio em função da temperatura e da umidade relativa do ar (Chen \& Jayas, 1998; Corrêa et al., 1998; Chen, 2000; Oliveira et al., 2004; Giner \& Gely, 2005; Resende et al., 2006; Francisco et al., 2007; Goneli, 2008; Bastos, 2009).
Ante a importância do conhecimento da higroscopicidade dos produtos agrícolas objetivou-se, neste trabalho, determinar as isotermas de dessorção das sementes de nabo forrageiro para diversas condições de temperatura e atividades de água e ajustar diferentes modelos matemáticos aos dados experimentais selecionando-se aquele que melhor representa o fenômeno obtido pelos métodos estático e dinâmico e ainda verificar a possibilidade de utilização de um único modelo para ambos os métodos.

\section{Material e MÉTODOs}

O trabalho foi desenvolvido no Laboratório de Pós-Colheita de Produtos Vegetais do Instituto Federal de Educação, Ciência e Tecnologia Goiano - IF Goiano, localizado no município de Rio Verde, GO. Para a condução do experimento foram utilizadas sementes de nabo forrageiro cultivado na área experimental da instituição, na entressafra 2009.

Foram utilizados dois métodos de determinação das isotermas de dessorção, quais sejam:

As sementes do nabo forrageiro foram colhidas manualmente com teor de água de aproximadamente $36 \%$ b.s. A dessorção do produto em camada delgada foi realizada para diferentes condições controladas de temperatura $\left(25,30,35\right.$ e $\left.40{ }^{\circ} \mathrm{C}\right) \mathrm{e}$ atividades de água entre 0,11 e 0,82 , até que o produto atingisse seu teor de água de equilíbrio com a condição do ar especificada.

As condições ambientais para realização dos testes de higroscopicidade foram fornecidas por meio de uma câmara condicionadora de atmosfera. As amostras, cada uma contendo $10 \mathrm{~g}$ de produto, foram envolvidas por um tecido permeável (tipo voile) para permitir a passagem do ar através das sementes e colocadas no interior do equipamento. A temperatura e a umidade relativa do ar foram monitoradas por meio de um psicrômetro instalado próximo às bandejas contendo as amostras.

Para determinação do teor de água de equilíbrio pelo método estático utilizou-se um lote de sementes colhidas mecanicamente com teor de água de $14,46 \%$ b.s. A dessorção do produto em camada delgada foi realizada para diferentes condições controladas de temperatura $\left(25,30,35\right.$ e $\left.40{ }^{\circ} \mathrm{C}\right) \mathrm{e}$ atividades de água entre 0,11 e 0,82 , até que o produto atingisse seu teor de água de equilíbrio com a condição do ar especificada.

As amostras, cada uma contendo $10 \mathrm{~g}$ de produto, foram envolvidas por um tecido permeável (tipo voile) para permitir a troca de vapor de água e colocadas no interior de dessecadores contendo soluções salinas saturadas.

As soluções salinas foram utilizadas para gerar ambientes de diferentes atividades de água (Tabela 1): cloreto de lítio $(\mathrm{LiCl})$, cloreto de cálcio $\left(\mathrm{CaCl}_{2}\right)$, nitrato de cálcio $\left(\mathrm{Ca}\left(\mathrm{NO}_{3}\right)_{2}\right)$, cloreto de sódio $(\mathrm{NaCl})$ e brometo de potássio $(\mathrm{KBr})$.

Os dessecadores com as amostras foram introduzidos em câmaras B.O.D. (Biochemical Oxygen Demand) mantidas nas diferentes temperaturas. A temperatura e a umidade relativa do ar no interior dos dessecadores foram monitoradas por meio de um data logger digital.

Durante as determinações pelos métodos dinâmico e estático as amostras foram pesadas periodicamente e o equilíbrio higroscópio das sementes foi atingido quando a 
Tabela 1. Atividade de água obtida pelas soluções salinas para diferentes temperaturas

\begin{tabular}{lcccc}
\hline \multirow{1}{*}{ Sal } & \multicolumn{4}{c}{ Temperatura $\left({ }^{\circ} \mathbf{C}\right)$} \\
\cline { 2 - 5 } $\mathrm{LiCl}$ & $\mathbf{2 5}$ & $\mathbf{3 0}$ & $\mathbf{3 5}$ & $\mathbf{4 0}$ \\
$\mathrm{CaCl}$ & 0,111 & 0,158 & 0,149 & 0,110 \\
$\mathrm{Ca}\left(\mathrm{NO}_{3}\right)_{2}$ & 0,307 & 0,307 & 0,217 & 0,207 \\
$\mathrm{NaCl}$ & 0,505 & 0,462 & 0,441 & 0,500 \\
$\mathrm{KBr}$ & 0,755 & 0,740 & 0,750 & 0,750 \\
$\mathrm{H}$ & 0,816 & - & 0,801 & 0,791 \\
\hline
\end{tabular}

massa permaneceu aproximadamente invariável durante três pesagens consecutivas.

Os teores de água das sementes de nabo forrageiro para cada método foram determinados por gravimetria utilizandose a estufa a $105 \pm 1{ }^{\circ} \mathrm{C}$, durante $24 \mathrm{~h}$, em duas repetições (Brasil, 2009).

Aos dados experimentais de teor de água de equilíbrio, obtidos por ambos os métodos, foram ajustados os modelos matemáticos frequentemente utilizados para representação da higroscopicidade de produtos agrícolas, cujas expressões estão apresentadas na Tabela 2.

Tabela 2. Modelos matemáticos utilizados para predizer o fenômeno de higroscopicidade de produtos agrícolas

\begin{tabular}{lll}
\hline \multicolumn{1}{c}{ Designação do modelo } & \multicolumn{1}{c}{ Modelo } \\
$\mathrm{Xe}=\exp \left\{\mathrm{a}-(\mathrm{b} \cdot \mathrm{T})+\left[\mathrm{c} \cdot \exp \left(\mathrm{a}_{\mathrm{w}}\right)\right]\right\}$ & Sigma Copace \\
$\mathrm{Xe}=\mathrm{a} \cdot\left(\mathrm{a}_{\mathrm{w}} \mathrm{b} / \mathrm{T}^{\mathrm{c}}\right)$ & Sabbah \\
$\mathrm{Xe}=(\mathrm{a}+\mathrm{bT}) /\left[\left(1-\mathrm{a}_{\mathrm{w}}\right) / \mathrm{a}_{\mathrm{w}}\right]^{1 / \mathrm{c}}$ & Oswin \\
$\mathrm{Xe}=\left[\operatorname{In}\left(1-\mathrm{a}_{\mathrm{w}}\right) /(-\mathrm{a} \cdot \mathrm{T}+273,16)\right]^{1 / \mathrm{c}}$ & Henderson \\
$\mathrm{Xe}=\left[\exp (\mathrm{a}-\mathrm{b} \cdot \mathrm{T}) /-\operatorname{In}\left(\mathrm{a}_{\mathrm{w}}\right)\right]^{1 / \mathrm{c}}$ & Halsey Modificado \\
$\mathrm{Xe}=\left(\mathrm{a} \cdot \mathrm{b} \cdot \mathrm{c} \cdot \mathrm{a}_{\mathrm{w}}\right) /\left[\left(1-\mathrm{c} \cdot \mathrm{a}_{\mathrm{w}}\right) \cdot\left(1-\mathrm{c} \cdot \mathrm{a}_{\mathrm{w}}+\mathrm{b} \cdot \mathrm{c} \cdot \mathrm{a}_{\mathrm{w}}\right)\right]$ \\
$\mathrm{Xe}=\mathrm{exp}\left[\mathrm{a}-(\mathrm{b} \cdot \mathrm{T})+\left(\mathrm{c} \cdot \mathrm{a}_{\mathrm{w}}\right)\right]$ & GAB \\
$\mathrm{Xe}=\mathrm{a}-\mathrm{b} \cdot \operatorname{In}\left[-(\mathrm{T}+\mathrm{c}) \cdot \operatorname{In}\left(\mathrm{a}_{\mathrm{w}}\right)\right]$ & Copace \\
$\mathrm{Xe}=\left\{1 /\left[\left(1-\mathrm{a}_{\mathrm{w}}\right) \cdot(1 / \mathrm{a} \cdot \mathrm{b}+((\mathrm{a}-1) / \mathrm{a} \cdot \mathrm{b}))\right]\right\}$ & Chung Pfost \\
\hline
\end{tabular}

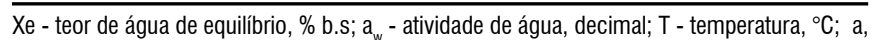
b, $\mathrm{c}$ - coeficientes que dependem do produto

Para o ajuste dos modelos matemáticos foi feita análise de regressão não linear, pelo método Gauss Newton, utilizando-se o Statistica 7.0. Para verificar o grau de ajuste de cada modelo considerou-se a significância do coeficiente de regressão pelo teste $t$ adotando-se o nível de $5 \%$ de significância, a magnitude do coeficiente de determinação $\left(\mathrm{R}^{2}\right)$, os valores do erro médio relativo $(\mathrm{P})$, do erro médio estimado (SE), o teste de Qui-quadrado $\left(\chi^{2}\right)$ a nível de significância de $1 \%$ e o intervalo de confiança a $99 \%(\mathrm{P}<0,01)$ além da verificação do comportamento da distribuição dos resíduos. Considerouse o valor do erro médio relativo inferior a $10 \%$ como um dos critérios para a seleção dos modelos, de acordo com Mohapatra \& Rao (2005). Os erros médios relativo e estimado e o teste de Qui-quadrado para cada um dos modelos, foram calculados conforme as seguintes expressões, respectivamente:

$$
\mathrm{P}=\frac{100}{\mathrm{n}} \sum \frac{|\mathrm{Y}-\hat{\mathrm{Y}}|}{\mathrm{Y}}
$$

$$
\begin{aligned}
\mathrm{SE} & =\sqrt{\frac{\sum(\mathrm{Y}-\hat{\mathrm{Y}})^{2}}{\mathrm{GLR}}} \\
\chi^{2} & =\sum \frac{(\mathrm{Y}-\hat{\mathrm{Y}})^{2}}{\mathrm{GLR}}
\end{aligned}
$$

em que:

Y - valor observado experimentalmente

Y - valor estimado pelo modelo

$\mathrm{N}$ - número de observações experimentais

GLR - graus de liberdade do modelo (número de observações menos o número de parâmetros do modelo)

Após a seleção do melhor modelo para a representação do teor de água de equilíbrio das sementes de nabo forrageiro obtido pelos métodos estático e dinâmico, foi feito o teste da hipótese de igualdade dos modelos utilizando-se a técnica de identidade de modelos descrita por Regazzi (2003) que permite analisar a equivalência entre os métodos utilizados.

Para obtenção das formas do modelo completo das equações com restrição, foram consideradas as variáveis dummy (D): sendo $D_{i}=1$ se a observação $Y_{i j}$ pertencer ao grupo i; e $D_{i}=0$, em caso contrário;

A regra de decisão baseou-se no teste de $\chi^{2}$ de acordo com a seguinte expressão:

$$
\chi_{\text {calculado }}^{2}=-\mathrm{N} \ln \left(\frac{\mathrm{SQR}_{\Omega}}{\mathrm{SQR}_{\mathrm{W}_{\mathrm{i}}}}\right)
$$

em que:

$\mathrm{N}$ - número de observações

$\mathrm{SQR}_{\Omega}$ - somatório dos quadrados residuais do modelo completo

$\mathrm{SQRW}_{\mathrm{i}}$ - somatório dos quadrados residuais do espaço paramétrico restrito

O valor tabelado de $\left(\chi^{2}\right)$ é função do nível de significância $\alpha$ e do número de graus de liberdade:

$$
\mathrm{v}=\mathrm{p}_{\Omega}-\mathrm{p}_{\mathrm{w}_{\mathrm{i}}}
$$

em que:

v - graus de liberdade do modelo

$\mathrm{P}_{\Omega} \quad$ - número de parâmetros do modelo completo

$\mathrm{Pw}_{\mathrm{i}}$ - número de parâmetros do modelo com restrição

\section{Resultados e Discussão}

As Tabelas 3 e 4 apresentam os parâmetros dos modelos ajustados aos valores de teor de água de equilíbrio higroscópico para as sementes de nabo forrageiro, obtidos por dessorção, pelo método dinâmico e estático para diferentes condições de temperatura e atividades de água.

Analisando os resultados apresentados nas Tabelas 3 e 4 , verifica-se que os modelos matemáticos utilizados para 
Tabela 3. Coeficientes dos modelos ajustados aos teores de água de equilíbrio higroscópico para as sementes de nabo forrageiro determinados pelo método dinâmico, com seus respectivos coeficientes de determinação $\left(\mathrm{R}^{2}\right)$, erros médios relativos (P), erros médios estimados (SE), Qui - quadrado $\left(\chi^{2}\right)$ e tendência de distribuição dos resíduos

\begin{tabular}{|c|c|c|c|c|c|c|c|c|}
\hline \multirow{2}{*}{ Modelo } & \multicolumn{3}{|c|}{ Coeficiente } & $\mathrm{R}^{2}$ & $\mathbf{P}$ & \multirow{2}{*}{$\begin{array}{c}\text { SE } \\
\text { (decimal) }\end{array}$} & \multirow{2}{*}{$\chi^{2}$} & \multirow{2}{*}{$\begin{array}{l}\text { Distribuição } \\
\text { dos resíduos }\end{array}$} \\
\hline & A & b & C & & & & & \\
\hline Sigma-Copace & $0,5267^{\star *}$ & $0,0172^{* *}$ & $1,0859^{* *}$ & 98,20 & 4,56 & 0,456 & 0,126 & Tendenciosa \\
\hline Sabbah & $2,7082^{\text {ns }}$ & $1,0996^{* *}$ & $-0,4496^{n s}$ & 73,07 & 15,86 & 6,846 & 1,899 & Tendenciosa \\
\hline Oswin & $9,2656^{* *}$ & $-0,1029^{* *}$ & $2,2821^{* *}$ & 98,21 & 4,45 & 0,456 & 0,126 & Tendenciosa \\
\hline Henderson & $0,0013^{\star *}$ & $8,1691^{\text {ns }}$ & $1,4611^{\star *}$ & 97,90 & 4,86 & 0,533 & 0,148 & Aleatória \\
\hline Halsey Modificado & $3,8257^{\star *}$ & $0,0321^{\star *}$ & $1,7894^{\star *}$ & 97,95 & 4,91 & 0,521 & 0,144 & Tendenciosa \\
\hline $\mathrm{GAB}$ & $4,1873^{\star}$ & $8,2798^{\text {ns }}$ & $0,8012^{\star \star}$ & 91,48 & 9,79 & 2,165 & 0,600 & Tendenciosa \\
\hline Copace & $1,3350^{* *}$ & $0,0170^{* *}$ & $1,9793^{* *}$ & 98,06 & 4,41 & 0,493 & 0,137 & Aleatória \\
\hline Chung Pfost & $18,9576^{\star *}$ & $4,0567^{\star *}$ & $2,6040^{\text {ns }}$ & 98,31 & 4,93 & 0,429 & 0,119 & Tendenciosa \\
\hline BET & $-179,070^{* *}$ & $0,4010^{\star *}$ & - & 75,00 & 13,58 & 6,125 & 1,637 & Tendenciosa \\
\hline
\end{tabular}

*Significativo a 0,01 pelo teste t; "Significativo a 0,05 pelo teste $t$; ns Não significativo

Tabela 4. Coeficiente dos modelos ajustados aos teores de água de equilíbrio higroscópico para as sementes de nabo forrageiro determinados pelo método estático, com seus respectivos coeficientes de determinação $\left(R^{2}\right)$, erros médios relativos $(\mathrm{P})$, erros médios estimados $(\mathrm{SE})$, Qui - quadrado $\left(\chi^{2}\right)$ e tendência de distribuição dos resíduos

\begin{tabular}{|c|c|c|c|c|c|c|c|c|}
\hline \multirow{2}{*}{ Modelo } & \multicolumn{3}{|c|}{ Coeficiente } & $\overline{R^{2}}$ & $\mathbf{P}$ & \multirow{2}{*}{$\begin{array}{c}\text { SE } \\
\text { (decimal) }\end{array}$} & \multirow{2}{*}{$\chi^{2}$} & \multirow{2}{*}{$\begin{array}{l}\text { Distribuição } \\
\text { dos resíduos }\end{array}$} \\
\hline & A & b & C & & & & & \\
\hline Sigma-Copace & $0,2243^{*}$ & $0,2243^{*}$ & $0,2243^{*}$ & 98,96 & 5,10 & 0,495 & 0,124 & Aleatória \\
\hline Sabbah & $34,5837^{\text {ns }}$ & $34,5837^{\text {ns }}$ & $34,5837^{n s}$ & 93,75 & 13,79 & 2,974 & 0,743 & Tendenciosa \\
\hline Oswin & $8,0797^{\star *}$ & $8,0797^{\star *}$ & $8,0797^{* *}$ & 98,41 & 5,52 & 0,757 & 0,189 & Aleatória \\
\hline Henderson & $0,0004^{*}$ & $0,0004^{*}$ & $0,0004^{*}$ & 97,02 & 8,91 & 1,399 & 0,350 & Tendenciosa \\
\hline Halsey Modificado & $3,2669^{* *}$ & $3,2669^{\star *}$ & $3,2669^{* *}$ & 98,68 & 5,08 & 0,630 & 0,157 & Aleatória \\
\hline $\mathrm{GAB}$ & $3,5312^{\star \star}$ & $3,5312^{\star \star}$ & $3,5312^{* \star}$ & 97,32 & 5,69 & 0,695 & 0,174 & Tendenciosa \\
\hline Copace & $1,1696^{* *}$ & $0,0096^{* *}$ & $1,9231^{* *}$ & 98,88 & 5,34 & 0,535 & 0,134 & Aleatória \\
\hline Chung Pfost & $19,7609^{* *}$ & $19,7609^{* *}$ & $19,7609^{* *}$ & 97,03 & 9,50 & 1,412 & 0,353 & Tendenciosa \\
\hline BET & $262,3960^{\text {ns }}$ & $0,4130^{* *}$ & - & 91,38 & 13,81 & 3,980 & 0,965 & Tendenciosa \\
\hline
\end{tabular}

${ }^{\star *}$ Significativo a 0,01 pelo teste t; *Significativo a 0,05 pelo teste t; ns Não significativo

descrever a higroscopicidade do nabo forrageiro apresentaram, para a maioria dos seus coeficientes, significância de regressão a nível de $1 \%$ de significância pelo teste t. Além disto os modelos, exceto os de Sabbah, GAB e BET, exibiram elevados valores do coeficiente de determinação superiores a $97 \%$ que, de acordo com Madamba et al. (1996) indicam uma representação satisfatória do fenômeno em estudo. De acordo com esses pesquisadores a utilização do coeficiente de determinação como o único critério de avaliação para a seleção dos modelos não lineares não constitui um bom parâmetro para representação do fenômeno. Então, para uma análise mais detalhada, foram utilizados outros parâmetros estatísticos para respaldar a seleção do melhor modelo.

Analisando a Tabela 3 nota-se que os valores obtidos referentes ao erro médio estimado (SE) dos modelos Sigma Copace, Oswin, Henderson, Halsey Modificado, Copace e Chung Pfost, apresentaram menores valores quando comparados aos demais e, na Tabela 4, observa-se que os modelos que obtiveram menores valores foram Sigma Copace, Oswin, Halsey Modificado, GAB e Copace.

Para ambos os métodos de determinação os valores do erro médio relativo $(\mathrm{P})$ foram inferiores a $10 \%$, exceto para os modelos de Sabbah e BET, no método dinâmico. De acordo com Mohapatra \& Rao (2005) valores inferiores a 10\% indicam uma representação adequada do fenômeno estudado.

Em relação ao teste de Qui-quadrado $\left(\chi^{2}\right)$ verifica-se que os nove modelos analisados tanto para o método dinâmico como para o estático, se encontram no intervalo de confiança de $95 \%$. Entretanto e comparando a magnitude dos valores do teste de $\chi^{2}$ dos modelos ajustados aos dados do teor de água de equilíbrio higroscópico obtidos pelo método dinâmico, Tabela
3, somente os modelos de Sigma Copace, Oswin, Henderson, Halsey Modificado, Copace e Chung Pfost e, na Tabela 4, os modelos Sigma Copace, Oswin, Halsey Modificado, GAB e Copace apresentaram menores valores em comparação com os demais.

Um modelo é considerado aleatório se os valores residuais se encontrarem próximos da faixa horizontal, em torno de zero, e também quando não formarem figuras definidas, não indicando tendenciosidade dos resultados. Se apresentar distribuição tendenciosa, o modelo é considerado inadequado para representar o fenômeno em questão (Goneli, 2008). Assim, entre a série de modelos testados para o método dinâmico apenas os modelos de Henderson e Copace apresentaram distribuição aleatória dos resíduos e no método de determinação estático a distribuição dos resíduos foi aleatória para os modelos de Sigma Copace, Oswin, Halsey Modificado e Copace, indicando ajuste mais adequado aos dados experimentais.

Entre os modelos testados para o método dinâmico o de Copace exibiu elevado coeficiente de determinação $(98,06 \%)$ e baixos valores do erro médio relativo, erro médio estimado e magnitude dos valores de Qui-quadrado, tal como distribuição aleatória dos resíduos, sendo assim selecionado para predição do equilíbrio higroscópico das sementes de nabo forrageiro.

No método estático, porém, os modelos de Sigma Copace, Oswin, Halsey Modificado e Copace exibiram os maiores coeficientes de determinação e menores valores dos erros médios relativo e estimado, menor magnitude dos valores de Qui-quadrado e distribuição aleatória dos resíduos, apresentando-se como adequados para representação do equilíbrio higroscópico das sementes de nabo forrageiro neste método de determinação. 
Neste contexto e se avaliando os dois métodos de determinação do teor de água de equilíbrio higroscópico e segundo os parâmetros estatísticos avaliados, observa-se que o modelo de Copace é o que melhor se ajusta aos dados experimentais obtidos pelos métodos dinâmico e estático. Corrêa et al. (1998) constataram, estudando o teor de água de equilíbrio de grãos de milho-pipoca, que o modelo de Copace foi o que melhor se ajustou aos seus dados experimentais.

A Figura 1 apresenta os valores experimentais do teor de água de equilíbrio das sementes de nabo forrageiro, obtidos por dessorção tal como suas isotermas calculadas pelo modelo de Copace, obtido pelo método dinâmico e estático.

\section{A.}

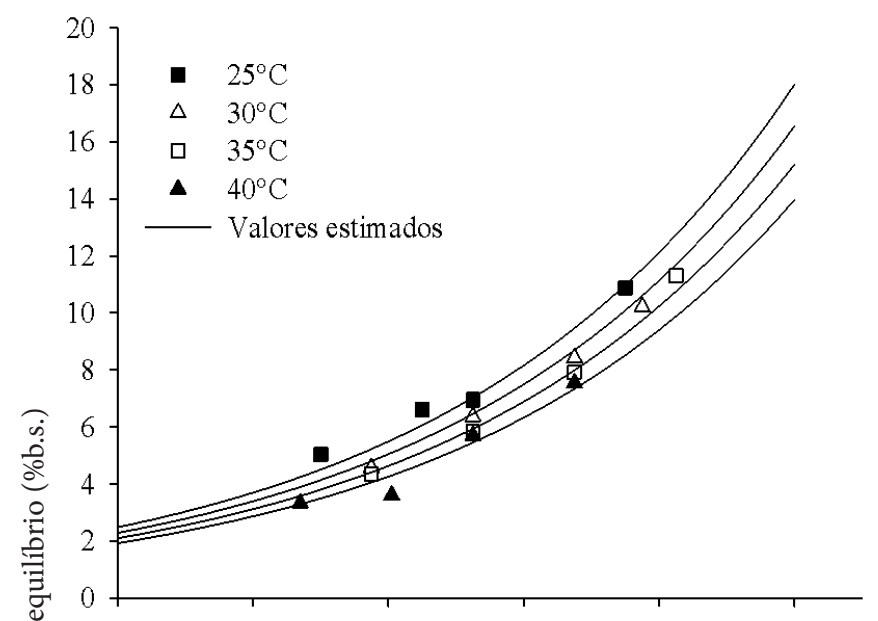

B.

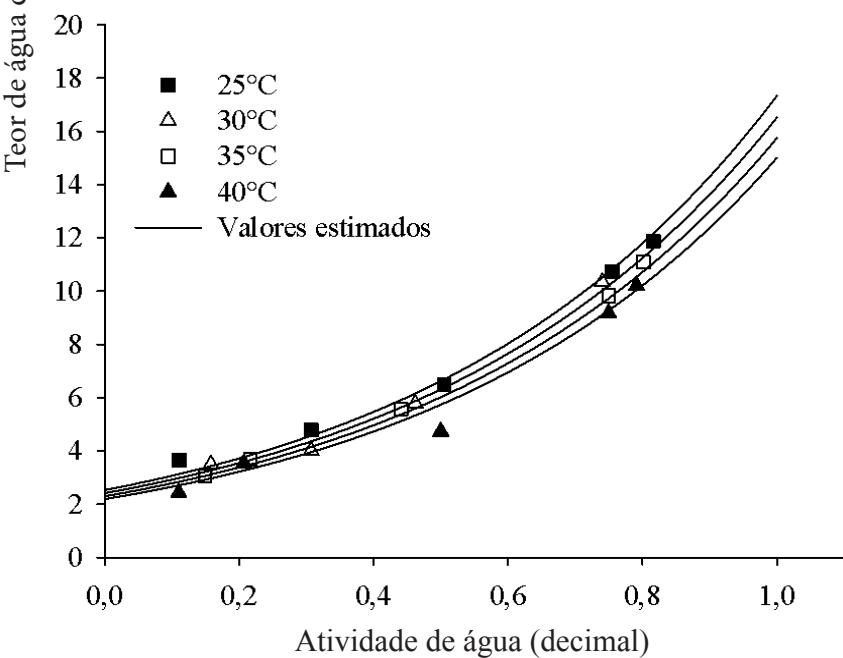

Figura 1. Valores experimentais de teor de água de equilíbrio higroscópico e isotermas de dessorção estimadas pelo modelo de Copace para as sementes de nabo forrageiro, obtidos pelo método dinâmico (A) e estático (B) em diferentes condições de temperatura e atividades de água

Sendo o modelo de Copace o que melhor se ajustou aos dados experimentais, procedeu-se ao teste de identidade de modelos objetivando-se viabilizar o uso de um único modelo para representação do equilíbrio higroscópico, independentemente da metodologia utilizada. Assim, os parâmetros do modelo de Copace, para o método estático $\left(\mathrm{a}_{1}, \mathrm{~b}_{1}\right.$ e c $\left.\mathrm{c}_{1}\right)$ e dinâmico $\left(\mathrm{a}_{2}, \mathrm{~b}_{2} \mathrm{e}\right.$ $c_{2}$ ), foram comparados entre si para verificar a sua igualdade. As seguintes hipóteses foram formuladas:

$$
\begin{aligned}
& \mathrm{H}_{\mathrm{o}}: \mathrm{a}_{1}=\mathrm{a}_{2} \text { e } \mathrm{b}_{1}=\mathrm{b}_{2} \text { e } \mathrm{c}_{1}=\mathrm{c}_{2} \\
& \text { versus }
\end{aligned}
$$

$\mathrm{H}_{\mathrm{a}}$ : existe pelo menos uma desigualdade entre os parâmetros Para o modelo de Copace obtêm-se as seguintes expressões: $\Omega$ - espaço paramétrico sem restrição ou modelo completo:

$$
\begin{aligned}
\mathrm{Y}_{\mathrm{ij}} & =\mathrm{D}_{1}\left\{\exp \left[\mathrm{a}_{1}-\left(\mathrm{b}_{1} \mathrm{~T}\right)+\left(\mathrm{c}_{1} \mathrm{a}_{\mathrm{w}}\right)\right]\right\}+ \\
& +\mathrm{D}_{2}\left\{\exp \left[\mathrm{a}_{2}-\left(\mathrm{b}_{2} \mathrm{~T}\right)+\left(\mathrm{c}_{2} \mathrm{a}_{\mathrm{w}}\right)\right]\right\}
\end{aligned}
$$

$\mathrm{W}$ - espaço paramétrico restrito por: $\mathrm{a}_{1}=\mathrm{a}_{2} \mathrm{e}_{1}=\mathrm{b}_{2}$ e $\mathrm{c}_{1}=\mathrm{c}_{2}$

$$
\begin{aligned}
\mathrm{Y}_{\mathrm{ij}} & =\mathrm{D}_{1}\left\{\exp \left[\mathrm{a}-(\mathrm{bT})+\left(\mathrm{ca}_{\mathrm{w}}\right)\right]\right\}+ \\
& +\mathrm{D}_{2}\left\{\exp \left[\mathrm{a}-(\mathrm{bT})+\left(\mathrm{ca}_{\mathrm{w}}\right)\right]\right\}
\end{aligned}
$$

A Tabela 5 apresenta as estimativas dos parâmetros do modelo completo $(\Omega)$ e do modelo com restrição (w) e as respectivas somas de quadrados residuais para o modelo de Copace.

A Tabela 6 mostra os resultados das hipóteses analisadas pelo teste de qui-quadrado.

Observa-se, na Tabela 6 , que o valor de $\chi_{\text {calculado }}^{2}$ foi inferior ao valor de $\chi_{\text {tabelado }}^{2}$. Assim, aceita-se a hipótese formulada $\left(\mathrm{H}_{\mathrm{o}}\right)$, ou seja, os modelos analisados para determinação do equilíbrio higroscópico das sementes de nabo forrageiro, pelos métodos estático e dinâmico, não diferem estatisticamente entre si, o que não ocorreu para Corrêa et al. (2005a) que, utilizando a técnica de identidade de modelos, que não foi possível ajustar um único modelo de Oswin aos dados experimentais da higroscopicidade de espigas de milho obtidos pelos métodos estático e dinâmico e para Resende et al. (2008) ao verificarem que não foi possível a utilização de um único modelo de Page para representar a secagem dos feijões preto e vermelho.

A Figura 2 apresenta os valores experimentais de teor de água de equilíbrio das sementes de nabo forrageiro, bem como suas isotermas, estimadas pelo modelo de Copace, utilizandose os mesmos coeficientes para os métodos de determinação dinâmico e estático.

Observa-se, na Figura 2, que as isotermas de dessorção das sementes de nabo forrageiro não se apresentaram na forma típica sigmoidal (tipo II) como observado para a maioria dos produtos agrícolas como, por exemplo, soja (Avira et al., 2004), trigo (Corrêa et al., 2005b), feijão (Resende et al., 2006), grão de bico, sementes de lentilhas, batata e pimentão verde (Furmaniak et al., 2007), arroz em casca (Basunia \& Abe, 2001; Iguaz \& Vírseda, 2007) e mamona (Goneli, 2008) classificadas como do tipo III (IUPAC, 1985). Tal comportamento indica que os principais constituintes do produto (solutos) apresentam pouca afinidade pelas moléculas de água, o que é perfeitamente explicável por ser o produto constituído prioritariamente de lipídios e carboidratos (Pena et al., 2010).

Verifica-se ainda que, para uma atividade de água constante, os valores de teor de água de equilíbrio higroscópico das sementes de nabo forrageiro diminuíram com o aumento da temperatura, seguindo a mesma tendência da maioria dos produtos agrícolas (Goneli, 2008). 
Tabela 5. Parâmetros do modelo completo $(\Omega)$ e do modelo com restrição $(w)$ e as respectivas somas de quadrados residuais para o modelo de Copace

\begin{tabular}{|c|c|c|c|c|c|c|c|c|c|c|}
\hline \multirow{2}{*}{$\begin{array}{l}\text { Estimativas } \\
\text { parâmetros }\end{array}$} & \multicolumn{9}{|c|}{ Parâmetros } & \multirow{2}{*}{$\operatorname{SQR}^{*}$} \\
\hline & $a_{1}$ & $\mathbf{b}_{1}$ & $c_{1}$ & $a_{2}$ & $b_{2}$ & $C_{2}$ & a & b & C & \\
\hline W & - & - & - & - & - & - & 1,2231 & 0,0123 & 1,954 & 5,0731 \\
\hline
\end{tabular}

${ }^{*}$ Soma dos quadrados residuais

Tabela 6. Teste das hipóteses $\left(\mathrm{H}_{\mathrm{o}}\right)$ utilizando-se o teste de qui-quadrado

\begin{tabular}{cccc}
\hline Modelo & GL $(v)$ & $\chi^{2}(0,01 ; 3)$ & $\chi_{\text {calculado }}^{2}$ \\
Copace & $6-3=3$ & 11,345 & $9,09^{\text {NS }}$ \\
\hline
\end{tabular}

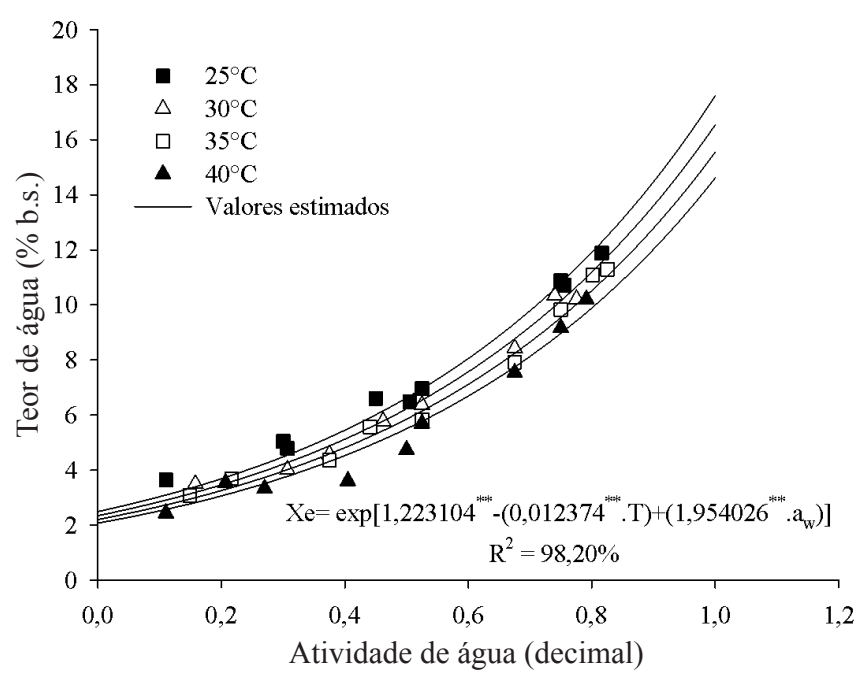

${ }^{*}$ Significativo a $1 \%$ pelo teste $t$

Figura 2. Valores experimentais e estimados pelo modelo único de Copace para o equilíbrio higroscópico das sementes de nabo forrageiro pelos métodos dinâmico e estático

\section{CONCLUSÕES}

1. O teor de água de equilíbrio higroscópico das sementes de nabo forrageiro é diretamente proporcional à atividade de água e decresce com o aumento de temperatura para um mesmo valor de atividade de água.

2. Para o método de determinação dinâmico o modelo de Copace é o que melhor representa a higroscopicidade das sementes de nabo forrageiro.

3. Para o método estático os modelos de Sigma Copace, Oswin, Halsey Modificado e Copace foram os que melhor representaram a higroscopicidade das sementes de nabo forrageiro, quando comparados com os demais modelos testados.

4. Segundo a técnica de identidade de modelos, é possível ajustar uma única equação de Copace aos dados experimentais para os dois métodos analisados.

\section{Agradecimentos}

Ao Conselho Nacional de Desenvolvimento Científico e Tecnológico (CNPq) e à Coordenação de Aperfeiçoamento de Pessoal de Nível Superior (CAPES) pelo apoio financeiro indispensável à execução do presente trabalho.

\section{Literatura Citada}

Araújo, L. F.; Corrêa, P. C.; Silva, R. F. Comparação de modelos matemáticos para descrição das curvas de dessorção de sementes de milho-doce. Pesquisa Agropecuária Brasileira, v.36, p.991-995, 2001.

Araújo, L. F.; Oliveira, L. S. C.; Perazzo Neto, A.; Alsina, O. L. S. L; Silva, F. L. H. da. Equilíbrio higroscópico da palma forrageira: Relação com a umidade ótima para fermentação sólida. Revista Brasileira de Engenharia Agrícola e Ambiental, v.9, p.379-384, 2005.

Avira, N. A.; Ajibola, O. O.; Oni, S. A. Sorption equilibrium and thermodynamic characteristics of soya bean. Biosystems Engineering, v.78, p.179-190, 2004.

Bastos, S. M. C. Propriedades termodinâmicas de adsorção de água de dois genótipos de arroz vermelho. Anápolis: UEG, 2009. 72p. Dissertação Mestrado

Basunia, M. A.; Abe, T. Moisture desorption isotherms of medium-grain rough rice. Journal of Stored Products Research, v.37, p.205-219, 2001.

Brasil. Ministério da Agricultura, Pecuária e Abastecimento. Secretaria Nacional de defesa Agropecuária. Regras para análise de sementes. Brasília: Mapa/ACS 2009. 398p.

Brooker, D. B.; Bakker-Arkema, F. W.; Hall, C. W. Drying and storage of grains and oilseeds. Westport: The AVI Publishing Company, 1992. 450p.

Chen, C. A rapid method to determine the sorption isotherms of peanuts. Journal Agricultural Engineering Research, v.75, p.401-408, 2000.

Chen, C.; Jayas, D. S. Evaluation of the GAB equation for the isotherms of agricultural products. Transactions of the ASAE, v.41, p.1755-1760, 1998.

Corrêa, P. C.; Goneli, A. L. D.; Resende, O.; Martinazzo, A. P.; Botelho, F. M. Comparação entre os métodos estático e dinâmico na determinação do equilíbrio higroscópico das espigas de milho. Revista Brasileira de Produtos Agroindustriais, v.7, p.153-161, 2005a.

Corrêa, P. C. Goneli, A. L. D. ; Resende, O. ; Ribeiro, D. M. Obtenção e modelagem das isotermas de dessorção e do calor esotérico de dessorção para grãos de trigo. Revista Brasileira de Produtos Agroindustriais, v.7, p.39-48, 2005 b.

Corrêa, P. C.; Martins, J. H.; Christ, D.; Mantovani, B. H. M. Curvas de dessorção e calor latente de vaporização para as sementes de milho pipoca (Zea mays). Revista Brasileira de Engenharia Agrícola e Ambiental, v.2, p.75-79, 1998.

Crusciol, C. A. C.; Cottica, R. L.; Lima, E. V.; Andreotti, M.; Moro, E.; Marcon, E. Persistência de palhada e liberação de nutrientes do nabo-forrageiro no plantio direto. Pesquisa Agropecuária Brasileira, v.40, p.161-168, 2005.

Derpsch, R.; Calegari, A. Plantas para adubação verde de inverno. Londrina: IAPAR, 1992. 80p. Circular, 73 
Domingos, A. K. Otimização da etanólise de óleo de Raphanus sativus L. e avaliação de sua estabilidade à oxidação. Curitiba: UFPR, 2005. 113p. Dissertação Mestrado

Francisco, F. G.; Usberti, R.; Toneli, J. T. C. L. Ajuste de isotermas de sorção de sementes de feijoeiro. Revista Brasileira de Sementes, v.29, p.35-39, 2007.

Furmaniak, S.; Terzyk, A. P.; Gauden, P. A. The general mechanism of water sorption on foodstuffs - Importance of the multitemperature fitting of data and the hierarchy of models. Journal of Food Engineering, v.82, p.528-535, 2007.

Giner, S. A.; Gely, M. C. Sorptional parameters of sunflower seeds of use in dryning and storage stability studies. Biosystems Engineering, v.92, p.217-227, 2005.

Goneli, A. L. D. Variação das propriedades físicas-mecânicas e da qualidade da mamona (Ricinus communis L.) durante a secagem e o armazenamento. Viçosa: UFV, 2008. 186p. Tese Doutorado

Hall, C. W. Drying and storage of agricultural crops. Westport: AVI, 1980. 381p.

Iguaz, A.; Vírseda, P. Moisture desorption isotherms of roung rice at high temperatures. Journal of Food Engineering, v.79, p.794-802, 2007.

IUPAC - International Union of Pure and Applied Chemistry. Reporting physisorption data for gas/solid systems. Pure and Applied Chemistry. v.57, p.603-619, 1985.
Madamba, P. S.; Driscoll, R.H.; Buckle, K. A. Thin-layer drying characteristics of garlic slices. Journal of Food Engineering, v.29, p.75-97, 1996.

Mohapatra, D.; Rao, P. S. A thin layer drying model of parboiled wheat. Journal of Food Engineering, v.66, p.513-518, 2005.

Oliveira, J. R.; Cavalcanti-Mata, M. E. R. M.; Duarte, M. E. M. Isotermas de dessorção de grãos de feijão macassar verde (Vigna unguiculata (1.) Walpers), variedade sempreverde. Revista Brasileira de Produtos Agroindustriais, v.6, p.61-70, 2004.

Pena, R. S.; Mendonça, N. B.; Almeida, M. D. C. Comportamento higroscópico do açaí em pó. Revista Brasileira de Produtos Agroindustriais, v.12, p.153-161, 2010.

Regazzi, A. J. Teste para verificar a igualdade de parâmetros e a identidade de modelos de regressão não linear. Revista Ceres, v.50, p. 9-26, 2003.

Resende, O.; Corrêa, P. C; Goneli, A.; Botelho, F. M.; Rodrigues, S. Modelagem matemática do processo de secagem de duas variedades de feijão (Phaseolus vulgaris L.). Revista Brasileira de Produtos Agroindustriais, v.10, p.17-26, 2008.

Resende, O.; Corrêa, P. C; Goneli, A. L. D.; Ribeiro, D. M. Isotermas e calor isostérico de sorção do feijão. Revista Ciência e Tecnologia de Alimentos, v.26, p.626-631, 2006. 